\title{
Dossiers partagés par les stagiaires avec ou sans formateur à l'IUFM de La Réunion : Analyses des traces
}

\author{
Jean SIMON, Jean-Paul GERARD, Claudine THEVENIN [ERTé \\ CALICO, Groupe GRRAPELI, IUFM de La Réunion]
}

- RÉSUMÉ : L'IUFM de La Réunion utilise depuis septembre 2005 une plate-forme de TCAO (BSCW). Nous analysons, dans cet article, le comportement et les actions des professeurs des écoles stagiaires sur cette plate-forme en distinguant selon qu'ils sont dans des groupes constitués uniquement de pairs ou dans des groupes constitués de pairs et de formateurs. Nous voulons observer si leur participation diffère selon qu'elle est plus ou moins imposée. Cette analyse est faite à partir des traces laissées sur la plate-forme. Nous utilisons comme unité d'analyse le "dossier partagé de plus haut niveau" (dpphn) dont nous expliquons la genèse et donnons la définition. Le dpphn révèle l'activité d'un groupe travaillant ensemble pour résoudre un problème. Dans la discussion, nous revenons sur les limites de la méthode employée et des résultats obtenus.

MOTS CLÉS : Méthodologie en analyse de traces, TCAO, Formation de formateurs, Théorie de l'Activité.

ABSTRACT : Since September 2005, the Teachers College of La Réunion uses a CSCW platform (BSCW). In this paper we analyze the behaviour and actions of the primary trainee on this platform according to the type of group they belong to: groups made up of only peers or groups made up of peers and trainers. The second type (including trainers) should be more restricted than the first. We wish to compare the peers' role and participation in each of these types of groups: free or restricted. This analysis is based on the traces left on the platform. In this paper, we also offer a definition of the "higher level shared folder" which is used as the unit of analysis. Such an entity reflects the activity of a group working jointly to solve a problem. In the discussion, we reconsider the limits of the method employed and the results obtained.

KEYWORDS : Methodology in trace analysis, CSCW, Teachers training, Activity Theory.

- 1 . Introduction

- 2 . Méthodologie

- $\underline{3 . \text { Résultats }}$

- 4 . Discussion

- 5 . Conclusion

- BIBLIOGRAPHIE

\section{Introduction}

\subsection{Le contexte}

L'Institut Universitaire de Formation des Maîtres (IUFM) de la Réunion forme des étudiants et des stagiaires au métier d'enseignant sur deux ans. De manière schématique, en première année, il s'agit de préparer les étudiants au concours qui leur permettra ensuite d'enseigner, tandis qu'en deuxième année, il s'agit de former les stagiaires au métier. On distingue les enseignants du $1^{\text {er }}$ degré (Professeurs des Ecoles, PE) de ceux du $2^{\text {nd }}$ degré (Professeurs des Lycées et Collèges, PLC, Professeurs des Lycées Professionnels, PLP). Dans cet article, nous nous intéresserons uniquement aux PE2, professeurs des écoles stagiaires de deuxième année. Leur formation fait alterner durant l'année des périodes où ils suivent des cours à l'IUFM et des périodes de stage dans les écoles où ils ont une classe en charge.

\subsection{Une plate-forme de TCAO : la demande}

Une plate-forme de Travail Collaboratif Assisté par Ordinateur a été mise en place en mai 2005 pour être opérationnelle en septembre de la même année. L'outil devait répondre à des demandes diverses émanant des 
PE2, des formateurs mais aussi du ministère. Si ces demandes convergeaient sur l'outil, elles n'en étaient pas pour autant identiques (Simon, 2006).

Pour les formateurs, la plate-forme devait permettre :

- de déposer des documents et servir de "mémoire collective" (mise en ligne des cours mais aussi des productions validées des stagiaires);

- d'améliorer des fiches de préparation de classe (dépôt sur le site d'une fiche de préparation puis remarques par le formateur ou par les collègues, amélioration de la fiche...);

- de faciliter la préparation de l'atelier d'analyse de pratique (plusieurs formateurs pouvant analyser la préparation du stagiaire);

- de mutualiser dans le cadre du mémoire (lorsque plusieurs stagiaires travaillent sur un même sujet) ;

- d'aider en ligne et à distance les stagiaires lors des stages.

Pour les stagiaires, l'outil devait permettre de mutualiser le travail de préparation de la classe lorsqu'ils étaient en stage.

Pour le ministère, l'outil devait permettre aux stagiaires d'apprendre à utiliser les TIC pour travailler ensemble (C2i2e $\underline{-})$.

\subsection{Une plate-forme de TCAO : le choix}

Auparavant, divers outils étaient utilisés par les formateurs et les PE pour répondre à leurs besoins : courriel, site Web, collecticiel du privé. Cette multiplicité des outils et leurs limites posaient problème car elles faisaient proliférer les formations nécessaires à leur prise en main, les espaces, les mots de passe... Il était donc nécessaire de trouver un outil unique qui réponde plus ou moins à l'ensemble de ces demandes. Le choix s'est alors porté sur BSCW (Basic Support for Cooperative Work) (Bentley et al., 1997). Les raisons qui ont présidé à ce choix étaient :

- BSCW est gratuit dans le cadre d'un usage éducatif,

- BSCW bénéficie d'une très bonne assistance technique par l'équipe du Fraunhofer FIT et

OrbiTeam et ceci toujours gratuitement,

- BSCW est utilisé par de nombreux organismes de formation d'enseignants:

- En France : IUFM de Bretagne, de Caen, ...

- À l'étranger (Hakkinen et al., 2003),

Et surtout, vu le polymorphisme de la demande, il y avait nécessité d'un logiciel qui laisse un degré de liberté suffisamment grand (Benali et al., 2002) pour y répondre et permettre notamment une coopération 2 aisée entre les différents usagers.

La contrepartie de ce degré important de liberté est que des spécifications quant à une utilisation optimale de l'outil n'existent pas (Sikkel et al., 2002). Cela amène les utilisateurs à devoir s'auto-organiser.

\subsection{Une plate-forme de TCAO : I'utilisation}

En une année scolaire, 2005-2006, 696 usagers sur les 1300 environ que comporte l'IUFMํㅡ, soit plus de la moitié de la population de l'IUFM, ont généré 396003 enregistrements sur la plate-forme qui allaient de la simple consultation à la création et au maintien d'espaces de travail partagés complexes.

Dans cet article, nous analysons ce phénomène. La méthode d'analyse employée sera décrite de telle manière qu'elle puisse être reprise éventuellement par d'autres (Henri et al., 2006).

\subsection{Motivation}

Une telle description n'est cependant utile que si elle permet de découvrir des régularités. Celles que nous voulons repérer ici doivent permettre de distinguer les dossiers qui sont partagés de manière totalement libre de ceux qui le sont de façon imposée.

Cette question d'une participation plus ou moins libre n'est pas triviale. Comme le souligne (Bruillard, 2007) : p. 8 "La rhétorique de l'intégration des technologies dans l'éducation et la formation cache mal un discours fortement prescriptif". Ainsi, pour passer le $\mathrm{C} 2 \mathrm{i} 2 \mathrm{e}$, on impose aux stagiaires d'utiliser un espace numérique de 
travail (ENT) ou a minima une plate-forme de TCAO. On peut alors s'interroger quant à la pertinence d'une telle utilisation si elle n'obéit qu'à la motivation uniquement extrinsèque de la certification. Nous voulons donc savoir si indépendamment du C2i2e ministériel et de la volonté des formateurs de l'IUFM, les stagiaires PE2 ont utilisé librement la plate-forme. Notre hypothèse est que les PE2 sont allés au-delà du cadre d'utilisation de la plate-forme prescrit par l'institution et les formateurs et ont employé celle-ci pour répondre à leurs besoins propres.

Une question sous-jacente qui nous intéresse aussi est de voir s'il est possible de caractériser les activités selon les personnes qui opèrent et leurs objectifs, et d'observer comment les acteurs s'emparent des occasions qui leurs sont offertes (Bruillard, 2007).

\section{Méthodologie}

Notre objectif est d'étudier l'activité des PE2 aux travers des traces qu'ils ont laissées sur la plate-forme. Dans un premier temps nous allons indiquer le cadre théorique dans lequel nous nous situons (la Théorie de l'activité), dans un deuxième temps les données élémentaires que nous allons analyser (les divers types de traces laissées sur la plate-forme) et, dans un troisième temps, l'unité d'analyse que nous utiliserons (le dossier partagé de plus haut niveau abrégé en $d p p h n)$, cette unité d'analyse étant une structuration préalable des données élémentaires qui répond au cadre théorique choisi. Dans un quatrième temps, nous donnerons une définition de ces $d p p h n$, dans un cinquième temps, nous préciserons les $d p p h n$ sélectionnés et nous terminerons, dans un sixième temps, sur les aspects techniques des $d p p h n$.

\subsection{Cadre conceptuel : la théorie de l'activité}

Le travail sur la plate-forme étant l'expression d'une activité, nous l'analysons en empruntant une partie de ses concepts à la Théorie de l'Activité4 (Engeström, 1987). C'est ainsi que nous avons pris comme support les triangles d'Engeström repris sur la figure 1. Ceux-ci ont une bonne capacité descriptive et permettent de saisir la plupart des aspects de l'activité (Lewis, 1998).

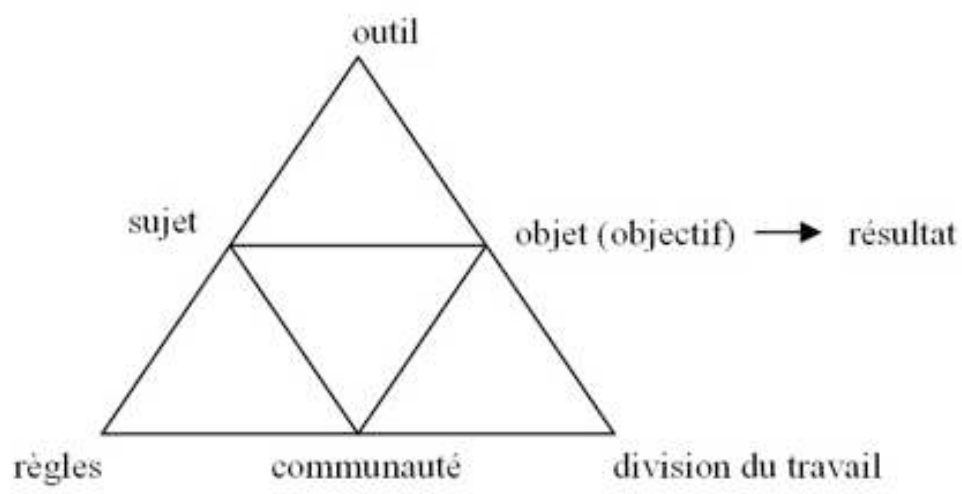

Figure 1・Structure de l'activité humaine (Engeström, 1987)

Dans l'activité, le sujet vise un objectif, qui débouche sur un résultat. Pour cela, il utilise des outils et agit au sein d'une communauté ou s'appuie sur celle-ci. Son rapport à cette communauté est défini par des règles. Pour atteindre le but, il peut être nécessaire de mettre en place une division du travail au sein de la communauté. Par exemple, dans le cadre de la chasse, on aura les chasseurs et les rabatteurs (Engeström, 1987).

Si nous utilisons ces triangles pour étudier l'activité réelle au sein de l'IUFM, nous voyons que nous ne pouvons considérer l'activité globale de celui-ci mais que nous devons faire une première distinction en fonction de l'objectif et de la communauté qui en découle : entre les étudiants de première année qui préparent le concours et les stagiaires de deuxième année qui se préparent au métier. De la même façon, nous devrons ensuite faire des distinctions entre le $1^{\mathrm{er}}$ degré (professorat des écoles) et le $2^{\text {nd }}$ degré (professorat des lycées et collèges). Ce qui est vrai de l'activité réelle de l'IUFM l'est aussi pour son activité virtuelle sur la plate-forme. Si nous nous plaçons dans le cadre de la Théorie de l'activité, l'unité d'analyse ne peut être l'activité globale de la plate-forme mais une unité plus petite définie essentiellement par deux des six paramètres : la communauté et l'objectif.

Par ailleurs, dans la TA, il est envisagé trois niveaux dans l'activité humaine (Kuutti, 1996) : celui de l'activité globale proprement dite, celui de l'action, une activité se décomposant en diverses actions et celui de l'opération, une action se décomposant en plusieurs opérations de base. Kuutti propose, parmi d'autres exemples, l'activité "mener à bien une recherche". Cette activité fera appel, entre autres, aux actions "faire des recherches bibliographiques", "participer à des conférences", "écrire des articles", qui pourront à leur tour faire 
appel aux opérations "user de syllogismes", "choisir les mots justes". Ces niveaux ne sont pas étanches, par exemple, une action devenue routinière pourra être considérée comme une opération. De plus selon l'activité de départ, ce qui pourra relever du niveau "activité" dans une recherche, relèvera éventuellement du niveau "action" dans une autre. Nous reprenons ces points dans la section suivante.

\subsection{Analyse des traces}

La méthode que nous avons employée est l'analyse des traces conservées sur la plate-forme. Nous reviendrons dans la conclusion sur les limites de ce type de démarches.

L'analyse de traces sur BSCW n'est pas un domaine nouveau. En 2001, (Appelt, 2001) s'intéresse à différents types de requêtes (création de dossiers, de documents, de discussion, lecture,....) et affiche des pourcentages pour chacune d'elles en les distinguant par type d'utilisateurs : néophytes ou confirmés. L'objectif est de voir les requêtes les plus fréquemment utilisées.

En 2003 (Daradoumis et al., 2003) regroupent les événements en quatre catégories (création, modification, lecture et déplacement) et vérifient si tous les utilisateurs participent au travail collectif.

En 2005, (Gonzalès et al., 2005) montrent l'intérêt de l'utilisation de BSCW dans la formation d'enseignants. Les traces qu'ils exploitent sont le temps d'utilisation, le nombre et le type de requêtes (lecture, création de documents,....). Ils distinguent les centres de formation de Pologne, de Roumanie, d'Italie et d'Espagne.

Enfin, en 2005 toujours, (Schümmer et al., 2005) cherchent à connaître l'implication d'un sujet dans le travail du groupe en utilisant aussi le temps comme métrique dans le cadre d'une recherche spatio-temporelle.

Cette liste n'est pas exhaustive mais la démarche dans les articles est plus ou moins la même : les traces étudiées sont les événements et l'unité d'analyse est le contenu global de la plate-forme. Le choix d'une telle unité d'analyse est en en cohérence avec les objectifs propres à ces recherches.

Si on observe ces travaux au travers du filtre de la TA, on comprend pourquoi nous ne pouvons adopter la même démarche. En effet, il n'y est pas fait de réelles différences entre les objectifs qui pourraient se résumer à "utiliser la plate-forme". Il n'y est pas fait non plus de différences entre les communautés puisque tous les usagers sont rassemblés dans une même catégorie ou dans des catégories qui ne sont pas en référence avec un type d'activité. Par exemple, les catégories "utilisateurs néophytes" et "utilisateurs confirmés" (Appelt, 2001) ne sont pas des communautés au sens de la TA car leurs membres ne partagent pas des objectifs identiques. Comparer leurs activités revient à comparer deux rapports du sujet à l'outil indépendamment du type d'activité considérée. Il n'y a pas non plus d'étude de la façon selon laquelle les dossiers sont organisés. De ce fait, il est difficile d'interpréter les résultats obtenus et de voir comment les activités se sont structurées.

$\mathrm{Si}$ on reprend notre exemple de l'activité réelle au sein de l'IUFM, cela équivaudrait à considérer l'activité globale de celui-ci indépendamment des filières, du rôle des différents acteurs (formateurs, usagers), du type de cours (magistraux, travaux dirigés) de la distinction $1^{\text {er }}$ et $2^{\text {nd }}$ degré, ou de la distinction $1^{\text {ère }}$ et $2^{\text {ème }}$ année... Nous pourrions repérer, comme cela est fait dans ces articles, quelques régularités : coût, absentéisme, taux d'occupation des salles, nombre d'heures de cours, type de cours,... Mais ces régularités ne seraient pas exploitables car trop générales, puisque l'on sait que les coûts varient selon les filières, de même l'absentéisme selon les années, le type de cours selon les degrés ou le taux d'occupation des salles selon la période de l'année. Comme le signale (Kuutti, 1996) : p. 31, "the actions cannot be understood without a frame of reference created by the corresponding activities".

Dans ces recherches, le grain d'observation utilisé est trop gros. Pour répondre à nos questions, il nous faut un grain plus fin et, pour le définir, nous nous appuyons sur la TA.

\subsection{Unité d'analyse : le dossier partagé de plus haut niveau (dpphn)}

À partir du moment où nous considérons que nous ne pouvons prendre l'activité globale de la plate-forme comme unité d'analyse il nous faut en choisir une plus petite. Les traces que l'on peut trouver sur BSCW sont celles des éléments de l'ontologie proposée par (Prinz et Zaman, 2005), qui distinguent entre :

- les "objets" : BSCW Folder (dossier), BSCW Document (document), BSCW User (utilisateur), BSCW Workspace (espace de travail),...

- et les "événements" relatifs à ces objets (BSCW-Event) tels que : création, modification, lecture, annotation...

La question est de savoir parmi ces différentes traces, celles qui reflètent une activité (ou plutôt son résultat) au sens de la $T A$. Nous éliminons d'emblée les traces d'événements qui sont des reflets d'opérations. Les documents, eux, peuvent être considérés comme les traces de résultats d'opération ou d'action. Les utilisateurs ou leurs espaces de travail ne reflètent pas non plus une activité même si ces traces permettent de savoir à quelles 
activités ils ont pris part. Les seules traces qui peuvent donc refléter une activité sont les dossiers.

Dans BSCW, ces dossiers sont organisés hiérarchiquement en dossiers et sous-dossiers. Cela nous amène à faire un parallèle entre cette hiérarchie (dossier / sous-dossier) et celle de la TA (activité / action). Il reste alors à déterminer à quel niveau de la hiérarchie un dossier reflète l'activité. Notre proposition est de prendre le dossier le plus élevé dans la hiérarchie auquel est associé un groupe. Nous l'appelons le dossier partagé de plus haut niveau (que l'on abrégera en $d p p h n$ ). L'idée qui prévaut à ce choix est que les utilisateurs de BSCW lorsqu'ils cherchent à résoudre collectivement un problème, créent pour cela un dossier qu'ils partagent. Ainsi, le $d p p h n$ reflète l'activité des membres d'un groupe (communauté) travaillant ensemble pour résoudre un problème (objectif). Dans la hiérarchie de dossiers, on peut considérer que le $d p p h n$ est la réponse globale au problème (le dpphn est le résultat d'une activité) tandis qu'un de ses sous-dossiers apporte, pour sa part, une réponse à une partie de ce problème (un sous-dossier est le résultat d'une action).

$\mathrm{Si}$ on revient sur les triangles d'Engeström, on peut alors considérer que cette structuration du $d p p h n$ en sous-dossiers reflète en partie la division du travail. Par ailleurs, grâce à l'analyse des traces-événements associés au dpphn, on peut étudier le rôle que se donnent les utilisateurs, ce qui constitue un autre aspect de la division du travail. Enfin, on peut, aussi, évaluer le respect des règles que se donnent ces communautés. On obtient ainsi sur la figure 2, la structuration de l'activité des PE2 sur la plate-forme vue au travers des triangles d'Engeström. L’objectif "être capable de faire classe" renvoie aux différentes demandes vues dans la section A.2.

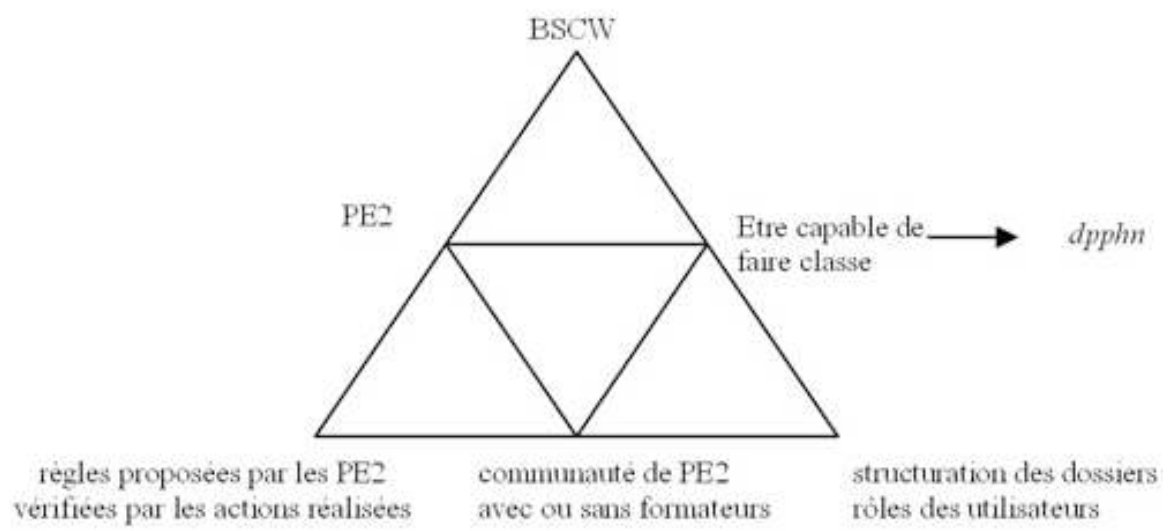

Figure 2 Application de la TA à notre situation

Dans le cadre de leur formation pour se préparer au métier d'enseignant, les PE2 utilisent BSCW pour créer des dpphn. On distingue ici les groupes de PE2 seuls, des groupes avec formateurs. On observe l'influence de cette distinction sur le respect de certaines règles ainsi que sur l'organisation des dossiers et les rôles que se donnent les usagers.

\subsection{Définition d'un dossier partagé de plus haut niveau (dpphn)}

Il y avait deux façons de définir les dpphn:

- restrictive : c'est un dossier partagé qui n'est le fils d'aucun dossier, autrement dit, qui n'est le sous-dossier d'aucun dossier ;

- large : c'est un dossier partagé qui n'est le descendant d'aucun dossier partagé mais qui peut être le descendant de dossiers non partagés et ceci sur plusieurs générations (ex : un dossier " $\mathrm{d}_{3}$ " est un dpphn parce qu'il est partagé et qu'il est le fils d'un dossier " $\mathrm{d}_{2}$ " non partagé et qui est lui-même le fils d'un dossier " $d_{1}$ " à la racine toujours non partagé).

Nous avons opté pour un compromis, un dpphn est :

- soit un dossier partagé fils d'aucun dossier, autrement dit un dossier partagé qui est à la racine et qui n'est donc contenu dans aucun autre dossier,

- soit un dossier partagé fils d'un dossier qui n'est lui-même pas partagé et qui est à la racine (on s'arrête donc à la première génération).

Justification de ce choix : les formateurs ont parfois regroupé dans un même dossier (non partagé) les dossiers qu'ils partageaient avec plusieurs groupes différents mais de même type (exemple : les dossiers partagés avec les 
différents groupes de PE2 sont regroupés dans l'espace de travail du formateur dans un seul dossier baptisé "PE2" qui lui n'est pas partagé).

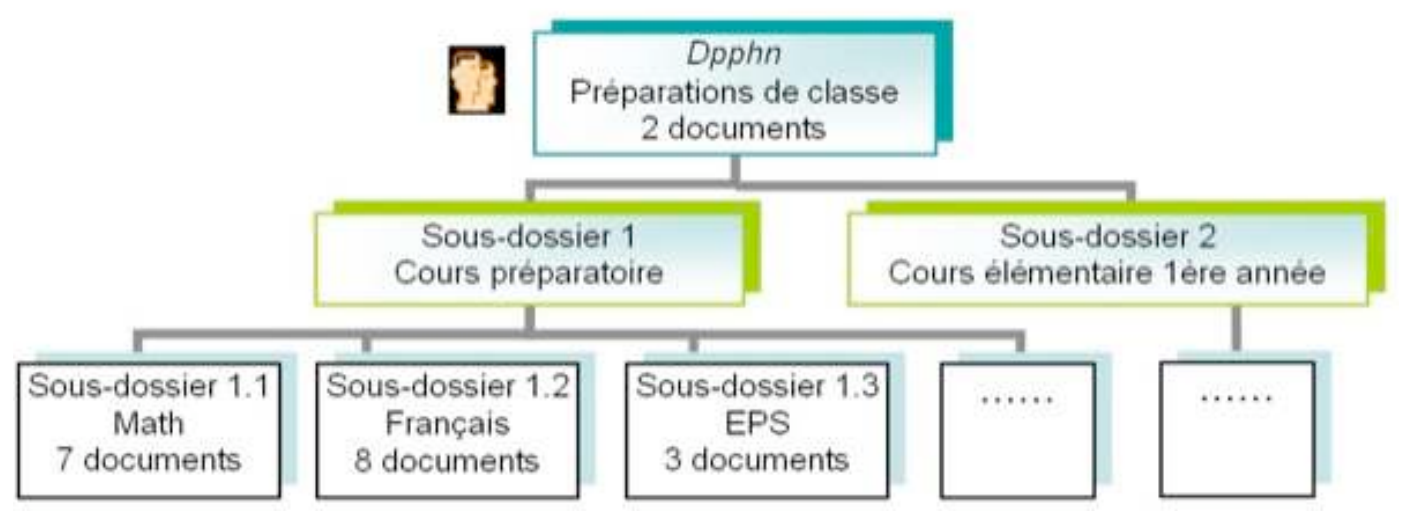

Figure 3 - Un exemple de dpphn

Sur l'exemple de $d p p h n$ donné en figure 3, les têtes symbolisent le groupe de membres associés au $d p p h n$. On voit aussi que chaque dossier peut lui-même contenir des sous-dossiers et des documents, nécessairement partagés par tout le groupe.

Ainsi défini, le $d p p h n$ nous permet de faire les distinctions entre les groupes mais aussi entre les objectifs. Par ailleurs, les traitements dont il peut faire l'objet sont assez facilement automatisables.

\subsection{Les dpphn analysés}

Pour répondre aux questions posées dans la section 1.5, nous allons analyser les $d p p h n$ partagés par les PE2 sur la plate-forme au cours de l'année scolaire 2005-2006 ainsi que les actions qui s'y rapportent (création, lecture, écriture...).

Nous nous intéressons aux PE2 pour plusieurs raisons :

- ils étaient les seuls stagiaires à devoir passer le C2i2e cette année-là,

- ils constituent une population assez homogène avec le même objectif global : "être capable d'enseigner dans le premier degré",

- ils bénéficient donc de la même formation.

En 2005-2006, ils constituaient 343 usagers.

La recherche est comparative et distingue deux catégories de dpphn:

- les dpphn partagés avec un formateur,

- les dpphn partagés entre pairs uniquement.

En effet, on peut considérer que le fait qu'un stagiaire ait l'obligation d'utiliser BSCW pour obtenir le C2i2e, rend son adhésion à un dossier davantage contrainte lorsque ce dossier est aussi partagé par un formateur/évaluateur. Néanmoins, cela ne signifie pas que tout partage avec un formateur soit une contrainte. De la même manière, le fait que les stagiaires soient contraints d'assister au cours par l'institution, ne veut pas dire qu'ils vivent ces cours comme des contraintes.

\subsection{Aspects techniques}

Techniquement, les éléments qui sont analysés sont ceux que l'on retrouve dans l'ontologie proposée par (Prinz et Zaman, 2005) (voir 2.3 ci-dessus).

Pour cela, nous avons adopté la même démarche que dans (Gonzalès et al., 2005). BSCW stocke toutes ses données dans un fichier texte. Nous avons traduit ce fichier en tables que nous avons travaillées avec un SGBD relationnel. 
Lorsque nous avons mis en place BSCW, et pour en faciliter l'appropriation par les utilisateurs, nous leur avons demandé d'indiquer dans leur identifiant leur appartenance à l'IUFM. Ainsi l'identifiant des PE2 commence par "PE2" et celui des formateurs par "pr" (pour professeur). Cela a permis ensuite de voir comment étaient constitués les groupes rattachés à un même $d p p h n$.

\section{Résultats}

Comme nous l'avons indiqué dans la section 2.5, et pour répondre aux questions posées dans la section 1.5 , nous avons séparé les dpphn en 2 catégories dans ce qui suit :

- ceux partagés uniquement par les PE2, appelés par la suite dpphn "PE2 seuls",

- ceux partagés par les PE2 et les formateurs, appelés par la suite dpphn "PE2 + prof".

\subsection{Nombre de dpphn}

\begin{tabular}{|l|l|l|l|}
\hline \multirow{2}{*}{ Type de groupes } & \multicolumn{2}{|l|}{ dpphn partagés par } & \multirow{2}{*}{ Nombre de dpphn } \\
\cline { 2 - 3 } & PE2 & prof & \\
\hline PE2 + prof & $\mathrm{x}$ & $\mathrm{x}$ & 78 \\
\hline PE2 seuls & $\mathrm{x}$ & & 289 \\
\hline
\end{tabular}

Tableau 1 • Répartition des dpphn selon la composition des groupes associés

Il apparaît dans le tableau 1 que les PE2 ont utilisé librement l'outil car ils ont partagé 289 dossiers sans les formateurs. On peut supposer que ce partage entre PE2 seuls a pour objectif de préparer la classe au vu des intitulés des dossiers et en s'appuyant sur (Simon, 2006). Nous verrons plus loin, dans la section 3.4.1, combien de PE2 sur les 343 ont effectivement participé à ces $d p p h n$ "PE2 seuls" et nous nous interrogerons, dans la section 4.1.3, s'il y a ainsi acceptabilité (Tricot et al., 2003) de la plate-forme par ces PE2.

\subsection{Communauté, taille des groupes}

Dans (Simon, 2007) nous analysons plus finement la taille des groupes. Nous reprenons ici les principaux résultats en nous intéressant uniquement aux PE2.

\begin{tabular}{|l|l|l|}
\hline Taille des groupes & PE2 seuls & PE2+prof \\
\hline moyenne & 8,3 & 19,8 \\
\hline écart-type & 11,9 & 19,4 \\
\hline
\end{tabular}

Tableau 2 • Taille des groupes associés aux dpphn en nombre de membres

Dans le tableau 2, la taille des groupes correspond au nombre de membres du groupe. On voit qu'il y a une nette différence selon le type de $d p p h n$. La taille moyenne des groupes est plus grande pour les dpphn "PE2+prof". L'écart type nous a amenés à approfondir ce résultat en prenant comme pivot une taille de groupe de 13 membres.

\begin{tabular}{|l|l|l|}
\hline $\begin{array}{l}\text { pourcentages des groupes selon leur } \\
\text { taille (en nombre de membres) }\end{array}$ & PE2 seuls & PE2+prof \\
\hline$\%$ de groupes à 13 et moins & $82 \%$ & $57,69 \%$ \\
\hline$\%$ de groupes à plus de 13 & $18 \%$ & $42,31 \%$ \\
\hline
\end{tabular}

Tableau 3 - Pourcentages des dpphn selon la taille des groupes 
Cette limite de 13 membres est celle proposée par Anzieu et Martin (1968), cités dans (Faerber, 2002) pour les groupes restreints qui, selon ces auteurs, serait encore appropriée au développement de la coopération. Avec $82 \%$ des tailles des groupes inférieures ou égales à 13, on constate alors que les $d p p h n$ "PE2 seuls" sont davantage dans le coopératif que les $d p p h n$ "PE2+prof". Les tailles des groupes associés aux $d p p h n$ "PE2+prof" semblent plus dispersées, un des groupes comptant jusqu'à 136 membres. Cependant, cette dispersion se comprend mieux si on se réfère aux objectifs visés par les formateurs (voir 1.2) qui sont beaucoup plus variés que ceux des PE2. Alors que les $d p p h n$ "PE2 seuls" servent essentiellement à mutualiser le travail de préparation de la classe, certains $d p p h n$ "PE2+ profs" sont utilisés, par exemple, pour le travail sur le mémoire qui laisse supposer des petits groupes et un travail collaboratif/coopératif, et d'autres pour la mise en ligne de cours qui concerne plusieurs groupes-classes de 27 PE2 en moyenne.

\subsection{Division du travail : la structuration des données}

Comme nous l'avons vu à la section 1.3, BSCW ne propose aucune ligne de conduite pour structurer les dossiers (Sikkel et al., 2002), si bien que c'est aux utilisateurs qu'il appartient de s'auto-organiser et de se répartir les tâches. Cette organisation est importante car elle doit permettre de diminuer la masse de travail pour le groupe en en réduisant la complexité et en permettant à chacun de s'y retrouver rapidement (Herrmann et al., 2004). (Nicol et al., 2005) ont montré que, selon le logiciel utilisé et selon le type d'activité envisagé, la structuration de cet archivage n'est pas la même. Pour (Prinz et Zaman, 2005), cette structuration est en général pauvre et les utilisateurs ont des difficultés pour repérer les documents.

Dans BSCW, l'organisation des $d p p h n$ est hiérarchique et se fait sur la base de dossiers et de sous-dossiers où sont rangés les documents (voir figure 3). Nous avons envisagé trois paramètres pour décrire l'organisation d'un dpphn (voir (Simon, 2007) pour une description plus détaillée). Le premier est le nombre de documents à ranger, le second est le nombre de sous-dossiers et le troisième est le nombre de niveaux de sous-dossiers du dpphn.

\begin{tabular}{|l|l|l|}
\hline & PE2 seuls & PE2+ prof \\
\hline $\begin{array}{l}\text { A: nb moyen de documents par } \\
\text { dpphn }\end{array}$ & 5,26 & 17,58 \\
\hline \hline $\begin{array}{l}\text { B: nb moyen de sous-dossiers par } \\
\text { dpphn }\end{array}$ & 0,64 & 4,52 \\
\hline ratio : A/(B+1) & 3,21 & 3,18 \\
\hline
\end{tabular}

Tableau 4 • Répartition des documents au sein des sous-dossiers dans les $\operatorname{dpphn}($ Le ratio se calcule avec $\mathrm{B}+1$ car le dpphn est lui aussi un dossier)

Comme le montre le tableau 4, les PE2 mettent peu de documents dans leurs $d p p h n$, ce qui les amène à créer peu de sous-dossiers par dpphn : tous les documents sont placés directement dans le dpphn. Les dpphn "PE2+prof" contenant trois fois plus de documents en moyenne, il y a de ce fait davantage de sous-dossiers créés. Par ailleurs, il est intéressant de voir que le ratio nombre de documents dans nombre de dossiers est de 3 environ dans les deux cas. En reprenant la même étude sur l'année prochaine, nous verrons s'il peut s'agir d'une constante, ce qui est peu probable. Ce nombre de 3 documents étant très faible, on pourrait parler de "sur-organisation" (Simon, 2007).

Concernant le nombre de niveaux de sous-dossiers, 83,72 \% des dpphn PE2 seuls ne contiennent aucun sous-dossier et 77,61 \% des dpphn PE2+profs ont, au plus, un niveau de sous-dossier. Ce faible nombre de niveaux de sous-dossiers s'explique lui aussi par le faible nombre de documents (Simon, 2007).

\subsection{Division du travail : les rôles 5}

Nous avons indiqué dans la section 1.5, que nous voulions vérifier plusieurs points concernant l'utilisation de la plate-forme. Le premier est de savoir s'il existe des sujets qui appartiennent à des groupes contraints (avec formateur) et à aucun groupe non contraint. Ces sujets sont symptomatiques d'une participation obéissant à une motivation davantage extrinsèque. Un autre point concerne les règles proposées par les PE2 lors d'une enquête (Simon, 2006) qui, selon eux, doivent permettre le bon fonctionnement du TCAO.

Pour cela, nous devons au préalable, nous intéresser aux rôles que se donnent les usagers. Il faut distinguer, ici, entre ce type de rôles et les profils qui sont implémentés dans la machine (Herrmann et al., 2004). Ces derniers se définissent par un ensemble de droits concernant les manipulations que les usagers sont autorisés à faire sur la plate-forme : lecture, écriture, création de dossiers, création de documents, invitation de membres... Concernant 
ces profils, tous les usagers de l'IUFM sont inscrits sur la plate-forme et participent aux dossiers en tant que "membres". Autrement dit, ils ont tous les droits, hormis ceux réservés spécifiquement à l'administrateur.

Les rôles qui nous intéressent, donc, ne sont pas les profils implémentés dans BSCW mais les rôles que les usagers s'attribuent (Herrmann et al., 2004), ceux qu'ils se distribuent entre eux dans leur groupe de travail (Cheesman et Heilesen, 2001). Pour définir ces rôles (Herrmann et al., 2004) proposent quatre caractéristiques : la position, la fonction/tâche, les attentes de comportement (behaviour-expectations) et l'interaction sociale. Bien évidemment, ces caractéristiques sont fortement interconnectées ; par exemple, la position sera reliée aux fonctions. Pour notre part, nous nous sommes appuyés sur la seconde caractéristique (tâche/fonction) pour définir les rôles des usagers et nous proposons les cinq catégories suivantes :

- leader : crée au moins un dpphn,

- animateur : crée au moins un sous-dossier dans un $d p p h n$,

- producteur : dépose au moins un document dans un dpphn (ou dans un de ses sous-dossiers),

- lecteur : lit au moins un document,

- inactif.

Les termes de leader et d'animateur sont à prendre avec quelques précautions. En effet ce n'est pas parce qu'un utilisateur crée un $d p p h n$ ou un sous-dossier que c'est lui qui a lancé le groupe. On peut en effet imaginer que le groupe se soit constitué à l'initiative d'une autre personne qui a proposé de créer un $d p p h n$ et qu'ensuite les tâches aient été distribuées.

En nous appuyant sur cette catégorisation des rôles, nous avons analysé les deux types de $d p p h n$, "PE2 seuls" (tableau 5) et "PE2+profs" (tableau 6), en distinguant les différents types d'usagers : PE2 et formateurs. Bien évidemment, dans le tableau 5, nous ne retrouvons pas les formateurs.

\subsubsection{Les dpphn "PE2 seuls"}

\begin{tabular}{|l|l|l|l|}
\hline $\begin{array}{l}\text { Dans les dpphn"PE2 } \\
\text { seuls", }\end{array}$ & $\begin{array}{l}\text { Nombre } \\
\text { de PE2 }\end{array}$ & $\begin{array}{l}\% \text { par rapport } \\
\text { au nb total de } \\
\text { PE2 par rapport }\end{array}$ & $\begin{array}{l}\text { nb PE2 } \\
\text { membres }\end{array}$ \\
\hline leader & 107 & $31,20 \%$ & $39,19 \%$ \\
\hline animateur & 119 & $34,69 \%$ & $43,59 \%$ \\
\hline producteur & 150 & $43,73 \%$ & $54,95 \%$ \\
\hline lecteur & 269 & $78,43 \%$ & $98,53 \%$ \\
\hline inactif & 4 & $1,16 \%$ & $1,46 \%$ \\
\hline $\begin{array}{l}\text { membre d'au moins un } \\
\text { dpphn }\end{array}$ & 273 & $79,59 \%$ & $100 \%$ \\
\hline nombre total de PE2 & 343 & $100 \%$ & \\
\hline
\end{tabular}

Tableau 5 • Répartition des rôles des PE2 dans les dpphn "PE2 seuls"

Le tableau 5 se lit de la façon suivante : 107 PE2 différents sont des leaders car ils ont créé au moins un dpphn. Par rapport au nombre total de PE2 de l'IUFM (343), cela représente 31,20\% de la population totale, par contre ramené au nombre total de (273) PE2 membres d'un dpphn de type "PE2 seuls", cela fait 39,19\%.

Il faut signaler qu'il n'y pas d'inclusion systématique entre les catégories. Par exemple, si tous les leaders sont aussi des animateurs, tous les animateurs ne sont pas obligatoirement des producteurs. En effet, nous avons analysé les $d p p h n$ en regroupant les catégories "animateur" et "producteur", celle-ci compte alors 159 individus distincts soit un peu plus que les 150 producteurs que l'on voit dans le tableau. Cela revient à dire que certains animateurs ne sont pas producteurs et réciproquement.

Le nombre d'inactifs se calcule ainsi : c'est la différence entre le nombre de membres et le nombre de lecteurs. Il y a ainsi quatre personnes (273-269). Comme il n'y pas inclusion systématique nous avons vérifié que ces quatre personnes n'avaient pas été non plus leader, producteur ou animateur. On peut supposer que ces quatre 
PE2 ont été invités à participer à des $d p p h n$ par leurs collègues mais que cela ne les a pas intéressés.

Ce premier tableau permet de confirmer la réponse esquissée dans la section 3.1 quant à l'acceptabilité de la plate-forme par les PE2 : 78,43 \% d'entre eux ont été membres d'un dpphn auquel ne participait pas de formateur. Autrement dit près de $80 \%$ des PE2 ont décidé sans contrainte (institutionnelle) que la plate-forme pourrait leur être utile et l'ont effectivement utilisée. Nous reviendrons sur ce point dans la discussion.

$\mathrm{Si}$ on prend comme référence le nombre de membres des $d p p h n$, on voit que près de $40 \%$ des PE2 sont des "leaders" (à l'origine d'un dpphn). Ainsi, parmi les 273 membres d'un dpphn "PE2 seuls", les leaders ne constituent pas une petite minorité de décideurs. Parmi ces leaders, certains n'ont créé qu'un seul $d p p h n$ tandis que d'autres en ont créés jusqu'à huit.

Par ailleurs, toujours en ne considérant que les membres, plus de $40 \%$ des PE2 ont été animateurs et plus de $50 \%$ ont été producteurs. Nous reviendrons sur ces points dans la section 3.5.1.

\subsection{2. les dpphn "PE2+prof"}

\begin{tabular}{|l|l|l|l|l|}
\hline $\begin{array}{l}\text { Dans les dpphn } \\
\text { "PE2+prof", }\end{array}$ & $\begin{array}{l}\text { Nombre } \\
\text { de PE2 }\end{array}$ & $\%$ & $\begin{array}{l}\text { Nombre } \\
\text { de Prof }\end{array}$ & $\%$ \\
\hline leader & 10 & $2,92 \%$ & 13 & $59,09 \%$ \\
\hline animateur & 61 & $17,78 \%$ & 13 & $59,09 \%$ \\
\hline producteur & 142 & $41,40 \%$ & 13 & $59,09 \%$ \\
\hline lecteur & 285 & $83,09 \%$ & 16 & $72,72 \%$ \\
\hline inactif & 58 & $16,91 \%$ & 6 & $27,27 \%$ \\
\hline $\begin{array}{l}\text { membre d'au moins un } \\
\text { dpphn }\end{array}$ & 343 & $100 \%$ & 22 & $100 \%$ \\
\hline
\end{tabular}

Tableau 6 - Rôles des différents utilisateurs dans les dpphn "PE2+prof"

Le tableau 6 se lit de la même manière que le 5. Ainsi, lorsque l'on écrit que 13 formateurs sont des leaders, il s'agit de 13 formateurs différents. Nous calculons de la même manière le nombre de PE2 inactifs qui s'élève à 58 soit $16,91 \%$ de la population (343 PE2) et de formateurs inactifs qui s'élève à 6 soit 27,27\% (des 22 formateurs).

\subsubsection{Rôle des PE2}

Concernant l'aspect "contraint" de la participation, on constate, premier point intéressant, que le nombre de lecteurs PE2 est différent de seulement $5 \%$ selon les types de dossiers, 78,43\% pour les dpphn "PE2 seuls" contre 83,09\% pour les $d p p h n$ "PE2+prof". Cela peut se comprendre car il n'y avait de réelle obligation que pour les PE2 souhaitant obtenir le C2i2e 6 . Pour les autres, cela dépendait de leur rapport à l'enseignant. Le second point intéressant, est que l'on voit que 10 PE2 ont créé des $d p p h n$ associant des formateurs. Cela a pu être fait à la demande des formateurs mais on peut supposer que les PE2 voient aussi les formateurs comme des personnes susceptibles de les aider. On peut, en résumé, considérer que la participation à un $d p p h n$ avec formateur peut être vécue par le stagiaire comme davantage contraignante que celle à un $d p p h n$ partagé uniquement par les pairs mais qu'en aucun cas elle n'est vécue comme une contrainte absolue puisque 16,91\% d'entre eux sont restés totalement inactifs.

Concernant les PE2 animateurs, on voit que le pourcentage ici $(17,78 \%)$ est nettement inférieur à celui des dpphn "PE2 seuls" (34,69\%). Cela est lié au fait qu'ils n'étaient pas seuls dans ces groupes et que les enseignants prenaient en charge une partie de la structuration des dossiers.

Par contre, on voit que le pourcentage de producteurs, ici (41,40\%), est peu inférieur à celui des $d p p h n$ "PE2 seuls" (43,73\%). On peut considérer qu'il n'y a pas de réelle différence entre les deux types de $d p p h n$ pour les PE2 dans ce rôle.

\subsubsection{Rôle des formateurs}

Concernant la participation des enseignants, on constate sans surprise que 13 sur 22 sont en même temps leaders, animateurs et producteurs. Ce sont les mêmes formateurs qui jouent les trois rôles. Non seulement, ils 
créent une partie des dpphn mais aussi ils les structurent et y placent des documents. Plus intéressant est le fait qu'ils soient 22 à être membres et que 16 ont été lecteurs. On peut faire l'hypothèse, qui demanderait à être vérifiée par une enquête, que neuf formateurs ont été invités par leurs collègues mais que seuls trois d'entre eux ont répondu à l'invitation, et en n'allant que lire.

\subsection{Les règles}

Une analyse des rôles permet de voir ce qu'il en est de l'usage de la plate-forme mais elle permet aussi d'aller plus loin et de vérifier si certaines règles ont été respectées. Dans (Simon, 2006), les stagiaires proposaient un ensemble de règles nécessaires selon eux à la réussite du travail collaboratif. Nous allons étudier le respect de trois de ces règles :

- la règle affirmant "tout le monde doit collaborer",

- la règle réclamant "d'avoir des retours sur les productions",

- la règle consistant à "éviter du travail aux autres".

\subsubsection{La règle "tout le monde doit collaborer"}

La règle "tout le monde doit collaborer" a été affirmée par tous les PE2 participant à l'enquête. L'idée sous-jacente à cette règle était celle du donnant-donnant. Certains PE2 proposaient même que tout nouveau candidat doive apporter quelque chose pour entrer dans le groupe.

Pour vérifier le respect de cette règle, nous avons comptabilisé les lecteurs qui n'avaient pas été en même temps producteur, animateur ou leader. Pour les $d p p h n$ "PE2 seuls", ils étaient 110, et, pour les $d p p h n$ "PE2+prof", 123. Cette catégorie d'usagers est notamment connue dans les forums de discussion où elle est baptisée "lurkers" (Cheesman et Heilesen, 2001), (Herrmann et al., 2004). Ce sont les personnes qui viennent voir sans s'impliquer. Dans le cadre de la mutualisation, elles sont considérées comme des personnes qui viennent puiser dans les ressources sans donner en contrepartie, ce qui est généralement mal perçu. Ici, selon le type de $d p p h n$, nous avons entre $32 \%$ et $36 \%$ de "lurkers" ce qui fait $1 / 3$ de la population. On voit donc que la première règle n'est pas respectée et on peut se demander comment le système évoluerait s'il se déroulait sur une période supérieure à un an.

\subsubsection{La règle "avoir des retours sur les productions"}

Les PE2 souhaitaient connaître l'usage qui était fait des documents qu'ils déposaient et donc obtenir un retour sur ceux-ci. Pour vérifier si cette règle était respectée, nous nous sommes intéressés à l'annotation des documents. En effet BSCW permet d'attacher une note à tout document. Une note n'est pas obligatoirement un retour mais son absence peut être indicative d'une absence de retour, d'un non respect de la règle.

\begin{tabular}{|l|l|l|}
\hline $\begin{array}{l}\text { pourcentage de documents } \\
\text { annotés }\end{array}$ & $\begin{array}{l}\text { dans les dpphn } \\
\text { "PE2 seuls" }\end{array}$ & $\begin{array}{l}\text { dans les } \\
\text { "PE2+prof" }\end{array}$ \\
\hline par les PE2 & $3,13 \%$ & $1,59 \%$ \\
\hline par les formateurs & & $5,05 \%$ \\
\hline total & $3,13 \%$ & $6,64 \%$ \\
\hline
\end{tabular}

Tableau 7 • Pourcentage de documents annotés dans les 2 types de $d p p h n$

Le tableau 7 montre qu'il y a globalement très peu d'annotations même si les documents dans les $d p p h n$ "PE2+prof" sont davantage annotés.

Pour les dpphn "PE2 seuls", cela peut s'expliquer par le fait que pour les PE2, l'objectif est surtout de mutualiser les préparations pour la classe lors des stages. Ces stages ayant une durée très courte (trois semaines) l'exploitation des annotations ne peut pas avoir lieu.

Pour les dpphn "PE2+prof", où les objectifs sont de différents ordres, on peut supposer que les retours se faisaient en présentiel et que ce sont surtout les dpphn ayant pour objectif "accompagnement en stage des PE2" qui nécessitaient un retour via la plate-forme pour répondre juste à temps (Thevenin et al., 2006).

\subsubsection{La règle "éviter du travail aux autres"}


La règle consistant à "éviter du travail aux autres" fait partie de l'essence même du TCAO. Si le TCAO fait augmenter la charge de travail individuel sans pour autant améliorer le résultat, il est peu probable que les usagers continuent de le pratiquer. Une première façon d'alléger la charge est dans la structuration des dossiers comme on l'a vu dans le 3.3. Une seconde façon est de donner le plus d'indications possibles sur les documents et les dossiers que l'on dépose sur la plate-forme pour éviter à l'utilisateur potentiel qui veut en connaître l'objectif de devoir l'ouvrir. Pour cela, l'animateur a la possibilité d'associer un descriptif au dossier qui en expose le contenu.

\begin{tabular}{|l|l|l|}
\hline & $\begin{array}{l}\text { dans les dpphn } \\
\text { "PE2 seuls" }\end{array}$ & $\begin{array}{l}\text { dans les } \\
\text { dpphn } \\
\text { "PE2+prof" }\end{array}$ \\
\hline $\begin{array}{l}\text { pourcentages de dossiers ayant un } \\
\text { descriptif }\end{array}$ & $36,66 \%$ & $39,93 \%$ \\
\hline
\end{tabular}

Tableau 8 - Pourcentages de dossiers ayant un descriptif selon le type de $d p p h n$

Comme l'indique le tableau 8, plus du tiers des dossiers font l'objet d'un descriptif et on ne voit pas de différence notable entre les deux types de $d p p h n$. Cela signifie qu'un peu moins des deux tiers des dossiers restent sans descriptif.

La règle "éviter un surplus de travail aux autres" en explicitant ce que l'on dépose sur la plate-forme ne semble donc pas respectée non plus mais ceci est à modérer car :

- l'explication peut s'être déroulée en présentiel,

- certains dossiers peuvent se passer de descriptifs car leur nom est suffisamment explicite?,

- certains dossiers peuvent être la réplication d'autres dossiers avec des objectifs similaires décrits seulement dans l'une des répliques (lorsqu'un PE2 crée un dossier "math au cycle 1" son contenu sera probablement similaire à un autre dossier "math au cycle 2").

\section{Discussion}

Dans cette section, nous revenons dans un premier temps sur les résultats et dans un second temps, nous discutons de la méthodologie employée.

\subsection{Résultats}

Notre hypothèse selon laquelle les PE2 sont allés au-delà du cadre d'utilisation de la plate-forme prescrit par l'institution et les formateurs et ont employé celle-ci pour répondre à leurs besoins propres est vérifiée. Nous avons 289 dpphn PE2 seuls contre 78 avec les enseignants.

\subsubsection{Utilisation de la plate-forme pour mutualiser?}

Pourquoi les PE2 seuls ont-ils créé des dpphn ? On peut supposer que, pour eux, cela répond à une demande sinon ils ne l'auraient pas fait. Dans ce sens la plate-forme leur est utile. Nous ne parlons pas ici "d'utilité" au sens précis de (Tricot et al., 2003), mais au sens commun de "dont l'emploi satisfait un besoin", besoin qui est ressenti par l'usager. La plupart des auteurs qui ont travaillé sur le TCAO dans le contexte de l'enseignement signalent cette nécessaire utilité. Pour (Docq et Daele, 2001) les étudiants négligent les outils qui ne leur sont pas utiles. Pour (Bazin, 2004) une nouvelle technologie doit rencontrer un besoin. (Harrari et Rinaudo, 2005) eux parlent de gain. Certains leur reprochent presque cette exigence d'utilité et parlent "d'utilitarisme" $\underline{\text { Turban, }}$ $\underline{2005)}$.

Se pose ainsi la question de la nature du besoin ressenti. En nous appuyant sur l'enquête préalable $\underline{\text { (Simon, }}$ $\underline{2006)}$, et concernant la population particulière des PE2, on peut émettre l'hypothèse qu'il s'agit d'un besoin de mutualiser -8 : mutualiser les préparations et les ressources pour faire classe. Cela va dans le sens de plusieurs auteurs selon lesquels la mutualisation est souhaitée par les stagiaires (Bruillard, 2007), (Ferone, 2006), (Harrari et Rinaudo, 2005). Cependant, ce besoin de mutualiser n'est pas vrai pour toute population. Dans le cadre d'une préparation au concours par exemple, les étudiants sont moins enclins à le faire (Bazin, 2004), (Simon, 2007). C'est pourquoi, des analyses basées sur des entretiens ou questionnaires systématiques sur une partie significative de la population des PE2 restent nécessaires pour l'attester de manière plus formelle.

Si tout le monde veut mutualiser, il y a cependant un déséquilibre dans la participation. Ainsi, si la majorité 
des PE2 sont des lecteurs (environ $80 \%$ d'entre eux), ce qui confirme les résultats de (Appelt, 2001), seulement $55 \%$ des PE2 sont des producteurs. Ce déséquilibre est signalé aussi dans d'autres travaux, notamment ceux de (Daradoumis et al., 2003), (Cheesman et Heilesen, 2001) et (Herrmann et al., 2004).

Cela paraît être une évidence que d'affirmer qu'il faut qu'un outil soit utile pour être utilisé. Cela n'est pas toujours vrai, en effet le fait que l'institution préconise l'usage de plate-forme peut en biaiser l'utilisation (Bruillard, 2007). Comme le disent (Harrari et Rinaudo, 2005) "l'injonction au travail collaboratif peut paraitre paradoxale". Pour (Ferone, 2006) : p. 236, "... on peut s'interroger sur le sens que les professeurs stagiaires accordent à ces dispositifs qui les amènent à communiquer à distance avec des collègues qu'ils côtoient presque quotidiennement." Cela amène parfois à des emplois qui interpellent les stagiaires de par leur côté factice (Harrari et Rinaudo, 2005).

C'est pourquoi nous avons voulu voir en quoi le fait que les formateurs interviennent dans des groupes modifiait le comportement de ceux-ci.

\subsubsection{Des comportements différents selon que les formateurs sont là ou non}

Le rôle des formateurs dans le fonctionnement les groupes de TCAO est reconnu par la plupart des auteurs (Ferone, 2006), (Harrari et Rinaudo, 2005), (Bazin, 2004), (Martinez et al., 2006)... Non seulement, et comme on s'y attend, bien souvent, ce sont les formateurs qui définissent l'objectif de l'activité, mais les formateurs peuvent aussi jouer sur la division du travail (Harrari et Rinaudo, 2005), la structuration des groupes (Martinez et al., 2006), l'établissement implicite ou explicite de règles et le respect de celles-ci (Ferone, 2006). Cela peut même avoir éventuellement des effets non désirés "un formateur qui domine les échanges dans une liste altère la spontanéité des échanges donc leur volume et leur sincérité" (Ferone, 2006).

Si l'étude des dpphn ne permet pas de confirmer ce dernier point, elle corrobore par contre les autres. Lorsqu'ils participent à des $d p p h n$, les formateurs sont majoritairement à l'origine de ceux-ci : sur les 78 dpphn "PE2+prof", seuls 10 ont été créés par des PE2 (voir 3.4.2).

Du point de vue de la division du travail, on constate que les dossiers "PE2+prof" sont plus profonds (davantage de sous-dossiers par $d p p h n$ ) et contiennent aussi plus de documents (voir 3.3). Ainsi, la structuration des données n'est pas la même, nous avons même parlé de "sur-organisation" dans le cas des $d p p h n$ "PE2 seuls". Par ailleurs, concernant les rôles, on vient de voir que les PE2 sont nettement moins nombreux à être leader mais on constate aussi que les PE2 sont moins nombreux à être animateurs dans les dpphn "PE2+prof" (61, voir 3.4.2) que dans les dpphn PE2 (119 voir 3.4.1). Les rôles d'organisateurs sont ainsi assumés par les formateurs. Par contre, au niveau de la production, les PE2 participent autant quelque soit le type de dossier (150 vs 142), de même pour la lecture des documents (269 vs 285).

Les groupes constitués sont de plus grande taille lorsqu'ils incluent des formateurs : $42 \%$ des groupes avec formateurs ont plus de 13 membres contre seulement $18 \%$ pour les groupes PE2 seuls (voir 3.2).

Ainsi la participation des formateurs aux $d p p h n$ modifie la division du travail et la taille des groupes. De plus, comme ils en sont souvent les leaders, on peut supposer que des $d p p h n$ dépassent la seule mutualisation et ciblent d'autres objectifs (voir 1.2). Par contre, étant donné que le nombre de "lurkers" est sensiblement le même dans les deux types de dossiers (voir 3.5.1), on peut considérer que la participation des formateurs n'est pas réellement contraignante (voir aussi 3.4.2.1).

\subsubsection{Acceptabilité de la plate-forme?}

Sur cette base, peut-on dire qu'il y a acceptabilité de la plate-forme par les PE2 au sens de (Tricot et al., 2003) ? $\mathrm{Si}$ on se réfère à l'évaluation de celle-ci telle qu'il la propose, nous envisageons "l'acceptabilité en termes d'adéquation aux : ...attente des apprenants,..." uniquement, ce qui réduit déjà le champ. Au vu des résultats présentés, nous pourrions supposer qu'effectivement la plateforme est acceptable en termes d'attente des PE2. Surtout que certains PE2 nous ont fait grief de ne pas pouvoir l'utiliser pour mutualiser par la suite lorsqu'ils quittent l'IUFM. Il convient cependant d'en discuter. En effet, le fait qu'un PE2 soit seulement lecteur d'un dpphn ne semble pas suffisant pour affirmer que celle-ci répond à ses attentes. Nous pourrions donc considérer qu'il n'y a acceptabilité que pour les PE2 à la fois lecteurs et producteurs (ou animateurs). La plate-forme serait alors un outil acceptable pour seulement $43 \%$ d'entre eux. Ce pourcentage baisserait encore si nous nous interrogions pour savoir à partir de quel nombre de documents lus ou déposés, nous pourrions parler d'acceptabilité. La moyenne de 19,27 documents lus par lecteur suffirait-elle pour parler d'acceptabilité ? Une fois encore, seuls des enquêtes et des entretiens permettraient de répondre à ces questions.

\subsubsection{Apports, limites et perspectives}

Ainsi, les apports et les limites de ces résultats sont en grande partie liés à la méthodologie employée et nous en discuterons dans la section suivante. Nous voudrions seulement signaler en quoi le travail mené ici se singularise d'autres recherches. D'abord nous avons pu traiter des données réelles, résultats de l'activité réelle 
des PE2. Ensuite, nous avons traité un nombre conséquent de données, plusieurs centaines de milliers d'opérations effectuées sur plusieurs milliers de documents, répartis dans plusieurs centaines de $d p p h n$ produits par plusieurs centaines de PE2. De ce fait, en réponse aux exigences portées par (Daradoumis et al., 2003), on peut supposer que nos résultats sont davantage concluants que ceux que l'on obtiendrait sur de petits groupes dans le cadre d'expériences ponctuelles (e.g. (Schümmer et al., 2005)). La validité de ces résultats est d'ailleurs confortée par leur similarité avec ceux de (Ferone, 2006) qui étudie le même type de population en métropole. Par ailleurs, cette convergence des résultats nous amène à penser que le contexte (l'insularité de La Réunion) intervient peu, mais cela est à vérifier.

Maintenant, nous souhaitons voir ce qu'il en est, de manière plus fine, de la coopération entre les usagers (Daradoumis et al., 2003), (Schümmer et al., 2005). Nous avons vu que tous les PE2 ne participaient pas. Mais qu'en est-il de ceux qui ont été acteurs? Y a-t-il eu collaboration entre eux ou se sont-ils contentés de juxtaposer leurs apports comme amènent à le penser les résultats du 3.5.2 et 3.5.3 ? Cela nous conduira à nous intéresser davantage à la négociation dans les groupes (Stahl, 2003) : "qui fait quoi ?", "comment s'organise-t-on ?". Cette négociation pouvant se faire en présentiel lorsque les stagiaires se retrouvent à l'IUFM, cela peut expliquer les résultats observés quant au non respect des règles "retour sur production" et "explication des contenus".

Cette question en soulève une autre. En effet, s'il est vrai que tout apprentissage collaboratif assisté par ordinateur relève nécessairement du travail collaboratif assisté par ordinateur, le travail collaboratif assisté par ordinateur n'implique pas obligatoirement apprentissage. C'est pourquoi nous souhaitons aussi étudier si le travail effectué par les PE2 sur la plate-forme influe sur leur formation, leurs apprentissages.

Ce qui nous amène, en dernier lieu, à la question : s'il n'y a pas eu collaboration faut-il essayer de la promouvoir lors de la formation initiale comme le proposent (Turban, 2005), (Ferone, 2006) et (Baron et Bruillard, 2006) ? Si oui, comment? Comment peut-on montrer aux PE2 que collaborer est utile sans tomber dans l'injonction paradoxale signalée plus haut?

Avant de continuer dans ce sens il est nécessaire de vérifier si la méthodologie employée ici est pertinente et suffisante.

\subsection{Méthodologie}

Pour effectuer les recherches, nous avons utilisé la technique de l'analyse des traces en prenant comme unité le dossier partagé de plus haut niveau (dpphn) que nous avons défini en nous servant des concepts de la Théorie de l'Activité. Nous revenons sur chacun de ces points.

\subsubsection{Utilisation de la Théorie de l'activité dans le cadre de l'analyse de traces}

Comme le signalent (Baron et Bruillard, 2006), l'utilisation de la théorie de l'activité dans le TCAO ou l'ACAO est fréquente. Préconisée par (Kuutti, 1996) pour l'étude du TCAO, elle est reprise par (Lewis, 1998) pour l'ACAO puis par (Docq et Daele, 2001), (Stahl, 2003), (Ferone, 2006)... Pour notre part nous nous sommes appuyés sur celle-ci parce que nous sommes convaincus que toute analyse d'une activité doit tenir compte des raisons/objectifs de cette activité. L'usage que nous en faisons, cependant, se distingue de ces recherches par le fait que nous l'avons employée pour l'analyse des traces, ce qui, à notre connaissance, n'avait pas encore été réalisé.

Parmi les différentes traces possibles, le $d p p h n$ est la seule, tout au moins sur BSCW, qui reflète l'activité d'un groupe essayant de résoudre un problème. De fait, il intègre tous les sommets des triangles d'Engeström et notamment deux d'entre eux : la communauté et l'objectif. Il permet d'analyser l'activité sur la plate-forme de manière beaucoup plus souple que de prendre cette activité globalement. Nous avons pu, ainsi, faire varier le paramètre "communauté", pour distinguer les deux types ("PE2 seuls" et "PE2+prof") et avons observé l'impact sur les règles et la division du travail. Le paramètre "objectif" nous a servi pour expliquer certaines variations dans ces résultats concernant notamment les dpphn "PE2+prof".

\subsubsection{Apports et perspectives}

Au sens de la TA, comme le dpphn reflète l'activité, il donne du sens aux actions (Kuutti, 1996). De ce fait, il permet d'analyser beaucoup plus rapidement et simplement certains problèmes et notamment celui de la participation effective des usagers en TCAO (Schümmer et al., 2005), (Daradoumis et al., 2003). Grâce au $d p p h n$, on peut étudier cette participation en fonction des groupes mais aussi des objectifs que se donnent ces groupes. On envisage ainsi, notamment sur les groupes "PE2+prof", de descendre plus profondément dans la recherche en distinguant entre la finalité que donnent les formateurs à chacune de leurs activités : un $d p p h n$ défini pour suivre les mémoires d'un groupe de stagiaires devrait nécessairement être différent d'un $d p p h n$ défini pour accompagner des PE2 en stage.

Tel qu'il est défini, le dpphn permet aussi d'accepter d'autres métriques telles que le temps (Schümmer et al., 
2005). L'année des PE2 est rythmée par les stages en responsabilité : comment ces périodes se reflètent-elles dans les dpphn ? Parmi nos perspectives de travail, nous souhaitons vérifier si les dpphn destinés à la mutualisation (PE2 seuls) sont beaucoup plus vivants durant ces périodes en évaluant la fréquence des actions des différents stagiaires.

Le $d p p h n$ permet aussi d'accepter l'analyse de contenu (Ferone, 2006). Il est ainsi possible pour chaque $d p p h n$ d'étudier les titres, mais aussi les descriptifs associés à chaque dossier et à chaque document déposé ainsi que les commentaires associés à ce document. Ce type d'analyse nous éclairerait en partie sur les objectifs poursuivis par le groupe.

Il permet, de plus, de circonscrire les réseaux dans le cadre d'une analyse des réseaux sociaux (Social Network Analysis) (Martinez et al., 2006), (Reffay et Chanier, 2003). D'emblée, il donne les objectifs de ces réseaux et permet de les comparer. À l'opposé, utiliser l'analyse des réseaux sociaux au sein d'un $d p p h n$ permettra de compléter les premières observations faites ici. Cela permettra notamment d'affiner les différents rôles que se donnent les usagers sur la plate-forme (leaders, animateurs,...).

Enfin, le $d p p h n$ est facilement automatisable et réutilisable. Il devrait donc permettre de comparer les résultats entre diverses plates-formes de TCAO (Henri et al., 2006), (Reffay et al., 2008). Pour notre part, nous allons l'utiliser pour étudier l'activité sur la plate-forme en 2006-2007, ce qui permettra de faire une analyse comparative avec les résultats présentés ici.

\subsubsection{Limites et prolongements \\ 4.2.3.1. Limites liées à l'analyse de traces}

Les limites constatées dans les résultats ne sont pas liées à la définition du dpphn ni à son utilisation comme unité d'analyse mais au fait qu'il relève de l'analyse de traces. On reproche à l'analyse des traces d'être trop à la surface des choses (Bruillard, 2007) : (Schümmer et al., 2005) : p. 568, "It is now widely acknowledged that activity reports provide a surface analysis of collaboration at best".

Ainsi si le dpphn reflète l'activité d'un groupe essayant de résoudre un problème, la trace qu'il représente ne nous informe pas sur le type de problème à résoudre et l'analyse de contenu proposée précédemment ne nous donnera qu'une information incomplète.

C'est pourquoi les résultats obtenus doivent être corroborés par d'autres types d'analyses (Bruillard, 2007) et il est proposé en général de la compléter par d'autres techniques. (Martinez et al., 2006) utilisent ce qu'ils appellent des mixed methods dans lesquelles l'analyse des traces est complétée par des observations, par des questionnaires, et par l'analyse des réseaux sociaux (Nurmela et al., 1999). (Hakkinen et al., 2003) proposent une approche multi-méthodes et suggèrent des complémentarités entre les démarches :

- approche théorique / approche par les données,

- approche qualitative / approche quantitative.

C'est ce que nous envisageons de faire notamment au travers d'entretiens semi-dirigés auprès des formateurs pour qu'ils nous expliquent quels étaient leurs objectifs.

\subsubsection{Généraliser la définition}

Par ailleurs, la définition du dpphn donnée ici est fortement dépendante du logiciel utilisé, c'est-à-dire BSCW, et de la façon selon laquelle il permet d'organiser les données. Néanmoins, cela n'est pas réellement un problème dans le sens où il est envisageable de trouver une définition équivalente pour chaque type de logiciel. C'est dans ce sens que nous allons travailler en proposant une définition plus générique du $d p p h n$. Ainsi, nous suggérons de limiter l'unité d'analyse à l'espace de travail partagé par un groupe pour un problème qu'il veut résoudre plutôt que d'englober toute l'activité tracée par la plate-forme. Dans le cadre d'un forum, l'unité pourrait être un fil de discussion plutôt que le forum complet.

\section{Conclusion}

Dans le cadre de la recherche faite ici, concernant le travail des PE2 sur la plate-forme, l'utilisation des $d p p h n$ a permis de montrer que les PE2 sont allés au-delà du cadre d'utilisation de la plate-forme prescrit par l'institution et les formateurs et ont employé celle-ci pour répondre à leurs besoins. Elle a permis de montrer aussi que, selon que des formateurs ont été associés ou non aux groupes de travail, la participation des PE2 n'était pas la même. Lorsque les PE2 sont seuls :

- ils sont davantage leaders ou animateurs, 
- ils créent beaucoup plus de dpphn,

- les groupes associés à ces dossiers sont de plus petite taille,

- les dpphn contiennent moins de documents et moins de sous-dossiers.

Le premier point est lié au fait que, lorsque des formateurs participent à des $d p p h n$, ce sont eux bien souvent qui sont à l'origine de leur création et de leur structuration. Les trois derniers points invitent à penser que les $d p p h n$ "PE2 seuls" constituent des réponses à des demandes plus limitées, plus ponctuelles, que les dpphn "PE2+prof".

1 C2i2e : Certificat Informatique et Internet niveau 2 Enseignement délivré par les IUFM qui atteste que le stagiaire est capable d'utiliser les TIC pour s'informer, se former et former ses élèves. En 05-06, tous les PE2 devaient le passer mais son obtention n'entrait pas dans la validation de leur année de stage.

2 Quand nous ne le précisons pas explicitement, le terme de coopération englobe la collaboration.

$\underline{3}$ L'IUFM de la Réunion forment 1200 stagiaires avec environ 120 formateurs ou formateurs associés (hors professeurs des écoles maîtres formateurs (PEMF)).

4 Par la suite, nous abrégeons la Théorie de l'activité en "TA" pour alléger la lecture.

$\underline{5}$ Cette section, ainsi que la section 4.1.1, prend en compte les remarques émises par Christophe Reffay que nous remercions ici.

6 De fait un peu moins de $50 \%$ des PE2 de l'IUFM ont obtenu le C2i2e cette année-là.

7 Notons de manière anecdotique que la longueur des noms des dossiers dans les deux types de $d p p h n$ sont en moyenne de 20 caractères (20,06 "PE2 seuls" et 20,15 "PE2+prof") et celles des descriptifs de 50 (49,12 "PE2 seuls et 50 "PE2+prof"). On constate donc qu'il n'y a pas non plus de différence entre les deux types de $d p p h n$ à ce niveau-là.

$\underline{8}$ Une autre réponse qui n'est pas la plus importante est que la plate-forme constitue pour eux un soutien moral (Simon, 2006), (Ferone, 2006). Par les échanges qui s'y passent, ils constatent qu'ils ne sont pas seuls à rencontrer certains problèmes.

\section{BIBLIOGRAPHIE}

APPELT W. (2001). What Groupware Functionality Do Users Really Use? Analysis of the Usage of the BSCW System. Ninth Euromicro Workshop on Parallel and Distributed Processing (PDP'01), Mantova, Italy, p. 337-341.

BAZIN J.-M. (2004). Usage de plate forme collaborative gratuite dans le cadre de la préparation au CAPES de Documentation, actes du colloque TICE 2004, Compiègne, France,. Disponible sur Internet à http://edutice.archives-ouvertes.fr/docs/00/02/74/41/PDF/Bazin.pdf (consulté le 12/6/8)

BARON G-L, BRUILLARD E. (2006). Quels apprentissages dans des communautés d'enseignants en ligne ? In DAELE A. \& CHARLIER B. (dir.). Comprendre les communautés virtuelles d'enseignants. Pratiques et recherches. , l'HARMATTAN, 305 p, Paris, p. 177 - 197.

BENALI K., BOURGUIN G., DAVID B., DERYCKE A., FERRARIS C. (2002). Collaboration / Coopération. Information-Interaction-Intelligence, Actes des deuxièmes Assises nationales du GDR I3, J. Le Maître (Ed.), Cépaduès Editions, p. 247-261.

BENTLEY R., APPELT W., BUSBACH U., HINRICHS E., KERR D., SIKKEL K., TREVOR J., WOETZEL G. (1997). Basic Support for Cooperative Work on the World Wide Web. International Journal of Human Computer Studies: Special issue on Novel Applications of the WWW, Spring 1997, Academic Press, Cambridge, vol. 46, nº6, p. 827-846.

BRUILLARD E. (2007). Travail et apprentissage collaboratif à distance dans l'enseignement supérieur. Eléments de réflexion. À paraître aux Presses Universitaires de Rennes, Disponible sur Internet à http://www.stef.ens-cachan.fr/annur/bruillard/Eb coop CREAD fin.pdf (consulté le 7/5/8)

CHEESMAN R., HEILESEN S.B. (2001). Using CSCW for problem-oriented teaching and learning in a net environment, Poster, Euro Conference on Computer Supported Collaborative Learning (Euro-CSCL'01), 22-24 March, Maastricht, Netherland.

DARADOUMIS T., XHAFA F., MARQUèS J.M. (2003). Is an 'Effective' Online Group Really Effective? Proceedings of the Spanish Workshop on Trabajo en Grupo y Aprendizaje Colaborativo: experiencias y perspectiva. November 11, Donostia, p. 75-82.

DOCQ F., DAELE A. (2001). Uses of ICT tools for CSCL: how do students make as their's own the designed 
environment? Proceedings of Euro Conference on Computer Supported Collaborative Learning (Euro-CSCL'01), March 22-24, Maastricht, Netherland, p. 197-204.

ENGESTRöM Y. (1987). Learning by expanding: An Activity-Theoretical Approach to Developmental Research. Orienta-Konsultit Oy.

FERONE G. (2006). Liste de discussion et identité professionnelle des enseignants en formation, JOurnées Communication et Apprentissage Instrumentés en Réseau (JOCAIR'06), Amiens, France. p. 235-257. Disponible sur Internet à

http://edutice.archives-ouvertes.fr/docs/00/13/81/83/PDF/13- Ferone.pdf (consulté le 12/6/8)

FAERBER R. (2002). Le groupe d'apprentissage en formation à distance : ses caractéristiques dans un environnement virtuel. Larose F. \& Karsenti T. (Eds), La place des TICE en formation initiale et continue à l'enseignement : bilan et perspectives. Sherbrooke : ed. CRP (Univ. de Sherbrooke), p. 99-128.

GONZáLEZ V.R., GARCíA DE LA SANTA A., GORGHIU G., GORGHIU L.M. (2005). BSCW as a support system for distance teacher training. Recent Research Developments in Learning Technologies, Proceedings of the Third International Conference on Multimedia \& ICT's in Education, vol. 2, p. 696-701.

HAKKINEN P., JARVELA S., MAKITALO K. (2003). Sharing perspective in virtual interaction. B. Wasson, S. Ludvigen, U. hoppe (Eds) Computer Support for Collaborative Learning: Designing for change in Networked Environments. Proceedings of CSCL 2003, Dordrecht: Kluwer Academic Publishers, p. 395-404.

HARRARI M., RINAUDO J-L. (2005). Stagiaires et tuteurs - Un difficile engagement ? Ressentis à propos d'un dispositif de travail collaboratif dans la formation des professeurs documentalistes à l'IUFM de Caen et l'IUFM de Rouen. Symposium, formation et nouveaux instruments de communication (Symfonic), Amiens, France. Disponible sur Internet à

http://www.dep.u-picardie.fr/sidir/articles/harrari rinaudo.htm (consulté le 15-6-8)

HENRI F., CHARLIER B., PERAYA D. (2006). Les forums de discussion en milieu éducatif, témoignage sur la pratique de recherche. JOurnées Communication et Apprentissage Instrumentés en Réseau (JOCAIR'06), Amiens, France. p. 13-26.

HERRMANN T., JAHNKE I., LOSER K.U. (2004). The Role Concept as a Basis for Designing Community Systems. Cooperative Systems Design, Scenario-Based Design of Collaborative Systems, F. Darses, R. Dieng, C. Simone \& M. Zacklad (Eds.), p. 163-178.

KUUTTI K. (1996). Activity Theory as a Potential Framework for Human-Computer Interaction Research. Context and consciousness: Activity theory and human computer interaction. B.A. Nardi (Ed.), MIT Press, Cambridge, MA. p. 17-44.

LEWIS R. (1998). Apprendre conjointement : une analyse, quelques expériences et un cadre de travail. Quatrième Colloque Hypermedia et Apprentisage, Poitiers, France, p. 11-28.

MART1NEZ A., DIMITRIADIS Y., GOMEZ E., JORR1N I., RUBIA B., MARCOS J.A. (2006). Studying participation networks in collaboration using mixed methods. International Journal of Computer-Supported Collaborative Learning, Springer New York. Vol. 1, n³, September, p. 383-408.

NICOL D., LITTLEJOHN A., GRIERSON H. (2005). The importance of structuring information and resources within shared workspaces during collaborative design learning. Open Learning, Vol. 20, $\mathrm{n}^{\circ} 1$ / February 2005, p. 31-49.

NURMELA K., LEHTINEN E., PALONEN T. (1999) Evaluating CSCL log files by Social Network Analysis. C. Hoadley (Ed.), Computer Support for Collaborative Learning, Proceedings of CSCL'99, Mahwah, NJ: Lawrence Erlbaum Associates, p. 434-442.

PRINZ W., ZAMAN B. (2005). Proactive Support for the Organization of Shared Workspaces Using Activity Patterns and Content Analysis. GROUP'05: 2005 International ACM SIGGROUP Conference on Supporting Group Work, Sanibel Island, Florida, USA, ACM Press, p. 246-255.

REFFAY C., CHANIER T. (2003). How social network analysis can help to measure cohesion in collaborative distance-learning. B. Wasson, S. Ludvigsen, \& U. Hoppe (Eds.), Computer Support for Collaborative Learning: Designing for change in Networked Environments. Proceedings of CSCL 2003, Dordrecht: Kluwer Academic Publishers, p. 343-352.

REFFAY C., CHANIER T., NORAS M., BETBEDER M.-L. (2008). Contribution à la structuration de corpus d'apprentissage pour un meilleur partage en recherche. Numéro spécial "Échanger pour apprendre en ligne" de la revue STICEF, Vol 15. http://www.sticef.org/

SIKKEL K., GOMMER L., VAN DER VEEN J. (2002).Using Shared Workspaces in Higher Education. Innovations in Education and Teaching International, Vol. 39, n 1 , January 01, 2002, p. 26-45.

SIMON J. (2006). Mutualiser entre pairs, Expressions, Saint Denis, n²7, p. 127-133. Disponible sur Internet http://www.reunion.iufm.fr/Recherche/Expressions/27/Simon2.pdf (consulté le 15-6-8)

SIMON J. (2007). Auto-organisation d'espaces de travail coopératif dans les formations d'enseignants à l'IUFM de la Réunion, Environnements Informatiques pour l'Apprentissage Humain (EIAH'07), Lausanne, Suisse, p. 197-202. Disponible sur Internet

http://hal.archives-ouvertes.fr/docs/00/16/15/02/PDF/22.pdf (consulté le 15-6-8) 
SCHüMMER T., STRIJBOS J-W, BERKEL T. (2005). A new direction for log file analysis in CSCL: Experiences with a spatio-temporal metric. Proceedings of the international conference on Computer Supported Collaborative Learning (CSCL'05), Taipei, Taiwan, p. 567-576.

STAHL G, Knowledge Negotiation in Asynchronous Learning Networks. Proceedings of the 36th Hawaii International Conference on System Sciences (HICSS'03), Hawaii, p. 32-41

Disponible sur Internet

http://ieeexplore.ieee.org/ie15/8360/26341/01173632.pdf?arnumber=1173632 (consulté le 15-6-8)

THEVENIN, C., GERARD J-P, SIMON J. (2006). Le juste à temps et le juste ce qu'il faut comme dynamiques de soutien à un accompagnement de stage professionnel scénarisé. Analyse d'une expérience. JOurnées Communication et Apprentissage Instrumentés en Réseau (JOCAIR'06), Amiens, France. p. 483-500. Disponible sur Internet http://edutice.archives-ouvertes.fr/docs/00/13/87/32/PDF/29- Thevenin Gerard Simon.pdf (consulté le 15-6-8)

TRICOT A ., PLEGAT-SOUTJIS F., CAMPS J-F, AMIEL A., LUTZ G., MORCILLO A. (2003). Utilité, utilisabilité, acceptabilité : interpréter les relations en trois dimensions de l'évaluation des EIAH. Environnements Informatiques pour l'Apprentissage Humain (EIAH'03), Strasbourg, France, p. 391-402.

TURBAN, J-M. (2005). Formation sur des réseaux professionnels Listes de diffusion pour enseignants du premier degré. Environnements Informatiques pour l'Apprentissage Humain (EIAH'05), Montpellier, France, p. 425-430.

\section{- À propos des auteurs}

Jean SIMON est Maître de Conférences en informatique à l'IUFM de La Réunion où il anime le groupe de recherche GRRAPELI (Groupe de Recherche Réunion sur l'APpprentissage en Ligne à l'Iufm). Il fait aussi partie de l'ERTé Calico pilotée par E.Bruillard. Ses recherches portent sur la mutualisation, le TCAO et la FAD dans le cadre de la formation des professeurs stagiaires. Il est également chargé de mission TICE à l'IUFM et correspondant C2i2e.

Adresse : IUFM de La Réunion, Groupe de recherche GRRAPELI, Allée des Aigues Marines, Bellepierre, 97487 Saint-Denis Cedex

Courriel : jean.simon@,reunion.iufm.fr

Jean Paul GERARD est professeur d'Education Physique et Sportive à l'IUFM de la Réunion, Docteur en Didactique des disciplines. Il fait partie du groupe de recherche GRRAPELI de l'Université de la Réunion. Il fait aussi partie de l'ERTé Calico pilotée par E.Bruillard. Ses recherches sont orientées vers la modélisation des activités physiques à des fins didactiques et de diffusion, mais également vers la compréhension des phénomènes de diffusion par l'utilisation d'environnements numériques.

Adresse : IUFM de La Réunion, Groupe de recherche GRRAPELI, Allée des Aigues Marines, Bellepierre, 97487 Saint-Denis Cedex

Courriel : $\underline{\text { Jean-Paul.Gerard } @ \text { reunion.iufm.fr }}$

Claudine THEVENIN est Conseillère pédagogique. Elle est titulaire d'un DESS Utilisation des technologies de l'information et de la communication pour l'éducation et la formation. Ses recherches portent sur l'accompagnement des professeurs des écoles lors des stages en situation par le biais d'une plate-forme de travail collaboratif pour leur apporter des réponses "juste à temps" aux problèmes qu'ils rencontrent.

Adresse : IUFM de La Réunion, Groupe de recherche GRRAPELI, Allée des Aigues Marines, Bellepierre, 97487 Saint-Denis Cedex

Courriel : $\underline{\text { Claudine.Thevenin@ac-reunion.fr }}$

Référence de l'article :

Jean SIMON, Jean-Paul GERARD, Claudine THEVENIN, Dossiers partagés par les stagiaires avec ou sans formateur à l'IUFM de La Réunion : Analyses des traces, Revue STICEF, Volume 15, 2008, ISSN : 1764-7223, mis en ligne le 16/10/2008, http://sticef.org

(C) Revue Sciences et Technologies de l'Information et de la Communication pour l'Éducation et la Formation, 2008 